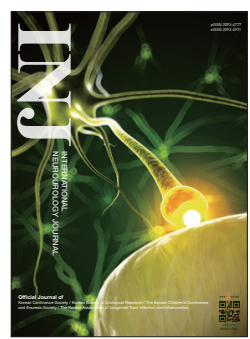

\title{
The Role of OnabotulinumtoxinA in Urology: What Is the Next Step?
}

\author{
Hong Sang Moon (ib http://orcid.org/0000-0003-2101-1019 \\ Associate Editor \\ Department of Urology, Hanyang University College of Medicine, Seoul, Korea \\ E-mail: moonuro@hanyang.ac.kr
}

Intradetrusor injection of onabotulinumtoxinA (BoTN-A) is an effective treatment method for patients with refractory overactive bladder $(\mathrm{OAB})$, with a favorable outcome after repeated injections. However, it has adverse effects and requires repeated periodic injections. Some of its adverse effects include hematuria, urinary tract infection, and increased postvoid residual volume [1]. Some patients are reluctant to undergo repeated BoTN-A injections leading to an increase in dropout rates.

The recommended dose of BoTN-A for patients with OAB is $100 \mathrm{U}$. It is administered at $5 \mathrm{U}$ per intradetrusor injection at 20 sites [2]. Decreasing the number of injections while simultaneously maintaining the same therapeutic effects would result in improved patient compliance. Liao et al. [3] reported the effects and safety of BoTN-A injection for patients with refractory $\mathrm{OAB}$ at different frequencies but at the same dose of $100 \mathrm{U}$. They administered 10, 20, and 40 intradetrusor injections of BoTN-A. The therapeutic and adverse effects were similar across the three frequencies. It would be promising to alleviate the pain associated with intradetrusor injection through local anesthesia.

Evaluation of the efficacy and adverse effects of intravesical instillation of liposome-encapsulated BoTN-A showed promising results [4]. While the liposome-encapsulated BoTN-A did not affect urinary urgency or incontinence, it decreased the severity of urgency experienced by patients with OAB. Thus, liposomes could be used as a carrier for delivering BoTN-A into the urothelium of patients with $\mathrm{OAB}$. It could be a promising and convenient treatment option for patients with refractory
$\mathrm{OAB}$ because it eliminates the need for intradetrusor injection and allows for easy repetition of BoTN-A treatment.

BoTN-A treatment can be used for the management of chronic pelvic pain (CPP). It decreases pelvic pain by reducing pelvic muscle spasm, central and peripheral nerve sensitization, and noxious neurotransmitter release leading to reduction of neurogenic inflammation [5]. It has been recently reported that BoTN-A yields good results in treatment of interstitial cystitis or bladder pain syndrome, pelvic floor muscle pain, and chronic prostatitis or CPP syndrome.

However, some BoTN-A treatment results were not satisfactory. Early treatment result of BoTN-A for benign prostatic hyperplasia showed promise but the results from long-term follow-up were unsatisfactory. We expect BoTN-A to be a probable candidate for the management of CPP owing to the unavailability of appropriate treatment option for this disease thus far; however, long-term follow-up is required.

- Conflict of Interest: No potential conflict of interest relevant to this article is reported.

\section{REFERENCES}

1. D’Ancona CA, Ferreira RS, Rassi MC. Botulinum toxin in neurogenic detrusor overactivity. Int Neurourol J 2012;16:139-43.

2. Liao $\mathrm{CH}$, Kuo HC. Practical aspects of botulinum toxin-A treatment in patients with overactive bladder syndrom. Int Neurourol J 2015;19:213-9. 
3. Liao CH, Chen SF, Kuo HC. Different number of intravesical onabotulinumtoxinA injections for patients with refractory detrusor overactivity do not affect treatment outcome: a prospective randomized comparative study. Neurourol Urodyn 2015 Apr 24 [Epub]. http://dx.doi.org/10.1002/nau.22780.

4. Chuang YC, Kaufmann JH, Chancellor DD, Chancellor MB, Kuo
HC. Bladder instillation of liposome encapsulated onabotulinumtoxina improves overactive bladder symptoms: a prospective, multicenter, double-blind, randomized trial. J Urol 2014;192:1743-9.

5. Jhang JF, Kuo HC. Novel treatment of chronic bladder pain syndrome and other pelvic pain disorders by onabotulinumtoxinA injection. Toxins (Basel) 2015;7:2232-50. 